\title{
Electroencephalographic patterns and clinical evolution in patients with encephalopathy in the intensive care unit.
}

\author{
Carballo, Karol Carolina; Carbonell Mateu, Ricardo; Peña, Susana; \\ Ayala Minero, Roberto Leonel
}

\author{
Karol Carolina Carballo \\ karolcarballo@gmail.com \\ Hospital Nacional Rosales, El Salvador \\ Ricardo Carbonell Mateu \\ Universidad “Dr. José Matías Delgado”, El Salvador \\ Susana Peña \\ Hospital Nacional Rosales, El Salvador \\ Roberto Leonel Ayala Minero \\ Hospital Nacional Psiquiátrico “Dr. José Molina \\ Martínez", El Salvador
}

\author{
Alerta \\ Ministerio de Salud, El Salvador \\ ISSN-e: 2617-5274 \\ Periodicidad: Semestral \\ vol. 2, núm. 2, 2019 \\ ralerta@salud.gob.sv
}

Recepción: 28 Mayo 2019

Aprobación: 26 Julio 2019

Publicación: 31 Julio 2019

URL: http://portal.amelica.org/ameli/journal/419/4191898010/

DOI: https://doi.org/10.5377/alerta.v2i2.8030

Forma recomendada de citar: Carballo KC, Carbonell Mateu R, Peña S, Ayala Minero RL. Patrones electroencefalográficos y evolución clínica en pacientes con encefalopatía en Unidad de Cuidados Intensivos. Alerta. 2019;2(2):153-162. DOI: 10.5377/ alerta.v2i 2.8030
Resumen: Introducción. En El Salvador, la encefalopatía es una de las condiciones clínicas con mayor incertidumbre al momento de valorar el pronóstico de un paciente en unidad de cuidados intensivos. El electroencefalograma ofrece un método de bajo costo, práctico, no invasivo y de alta disponibilidad mediante el cual se logra hacer una valoración del estado neurológico del paciente y podría contribuir al pronóstico. Objetivos. Describir los patrones electroencefalográficos y la evolución clínica de pacientes con encefalopatía ingresados en unidad de cuidados intensivos médica y quirúrgica durante los meses de septiembre a diciembre de 2018, en el Hospital Nacional Rosales. Metodología. Se realiza un estudio descriptivo de tipo serie de casos. Resultados. Se incluyeron pacientes con diagnóstico de encefalopatía y registraron variables clínicas, electroencefalográficas, edad, sexo, comorbilidades, causa de ingreso a unidad de cuidados intensivos médica o quirúrgica, uso de fármacos sedantes o relajantes, uso de aminas vasoactivas, estado de conciencia, complicaciones durante estancia intrahospitalaria, evolución y desenlace: defunción, traslado o alta. Conclusión. El patrón más frecuente entre los pacientes, con diferentes diagnósticos clínicos, fue el epileptiforme, mostrando el mayor grado de supervivencia entre ellos al ser tratados con fármacos antiepilépticos. La reactividad mostró tener valor pronóstico entre los pacientes. El 44,44\% de 9 pacientes no reactivos fallecieron, mientras que los 6 pacientes reactivos mostraron un $100 \%$ de supervivencia.

Palabras clave: Electroencefalografía, patologías encefálicas, pronóstico, cuidados intensivos, encefalopatías.

Abstract: Introduction. In El Salvador, encephalopathy is one of the clinical conditions with the greatest uncertainty when assessing the prognosis of a patient in the intensive care unit. The electroencephalogram offers a low cost, practical, non-invasive and highly available method through which an evaluation of the neurological status of the patient is achieved. Objective. Study was carried out with the aim of correlating electroencephalographic patterns and clinical evolution of patients with encephalopathy admitted to the medical and surgical intensive care unit during the months of September to 


\begin{abstract}
December 2018, in National Hospital Rosales. Methodology. An observational, descriptive, longitudinal, prospective. Results. Patients with a diagnosis of encephalopathy were included and chronicled clinical and electroencephalographic variables, age, sex, comorbidities, cause of admission to the intensive care unit, medical or surgical, use of sedative or relaxing drugs, use of vasoactive amines, state of consciousness, complications during intrahospital stay, evolution and outcome: death, transfer or discharge. Conclusion. The most common pattern among patients was epileptiform, showing the highest degree of survival among them, when treated with antiepileptic drugs. The reactivity showed a prognostic value among the patients, $44,44 \%$ of 9 non-reactive patients died, and while the 6 reactive patients showed a $100 \%$ survival of patients with encephalopathy and EEG pattern with reactivity present during the period of time admitted.
\end{abstract}

Keywords: Electroencephalography, encephalic pathologies, prognosis, intensive care, encephalopathies.

\title{
INTRODUCCIÓN
}

La encefalopatía es una condición clínica encefálica que se caracteriza por una disfunción cognitiva reflejada en cambios en la función o estructura cerebral. Esta es una de las disfunciones neurológicas más frecuentes que se presentan en el nivel hospitalario ${ }^{1}$, sobre todo en pacientes ingresados en unidades de cuidados intensivos. Se expresa con desorientación temporo-espacial, alteraciones en la memoria reciente, alucinaciones visuales, trastornos afectivos, alteración de ciclo vigilia sueño y alteraciones en el curso de los pensamientos con manía o delirio. Existen, además, tres anomalías motoras muy características de estos trastornos: el temblor, la asterixis y el mioclonus ${ }^{2}$.

En general, los síntomas que puede presentar cada paciente dependen de la causa de la encefalopatía, si es de tipo metabólica, hipertensiva, hepática, hipóxico isquémica, urémica, epiléptica, entre otras. Teniendo en común el daño cerebral y alteraciones de la conciencia en la escala de Glasgow ${ }^{3}$.

La encefalopatía hepática es el tipo de encefalopatía más común en pacientes ingresados en unidades de cuidados intensivos (UCI $)^{4}$, el cual es un síndrome que afecta principalmente a pacientes con hepatopatía crónica avanzada o derivación porto sistémico y se caracteriza por trastornos neuro-psiquiátricos ${ }^{5}$. Una de las formas más ampliamente utilizadas para valorar los grados de encefalopatía hepática son los criterios de WestHeaven $^{6}$. La causa más común de daño cerebral es la encefalopatía hipóxico isquémica, donde se induce la respuesta inflamatoria en el parénquima y además en la circulación sistémica. Posterior al evento se producen citosinas y leucocitos que son activados y migran hacia la lesión cerebral ${ }^{6}$.

La encefalopatía metabólica (ME) comprende todas aquellas alteraciones del sistema nervioso central (SNC) a partir de errores congénitos del metabolismo (ECM), metabolismo intermediario, enfermedades lisosomales y trastornos en la producción de energía ${ }^{7}$. En la encefalopatía epiléptica se considera que las descargas progresivas por epileptógenos evidentes en el electroencefalograma contribuyen a un progresivo deterioro de la función cerebral, acompañado de alteraciones motoras y mentales graves ${ }^{8}$.

Uno de los métodos diagnósticos que ofrece una opción económica y no invasiva para el diagnóstico de encefalopatías, es el electroencefalograma (EEG). Este se utiliza para registrar la actividad eléctrica cerebral en la superficie craneana, a través de electrodos. Esta actividad de las neuronas ocasiona potenciales postsinápticos excitatorios o inhibitorios, así como potenciales de acción que se transmiten de neurona 
a neurona, registrado en forma de trazos que reflejan la simetría y sincrónica del ritmo de fondo, la variabilidad de la actividad y la reactividad a determinadas maniobras de activación ${ }^{9}$. Este método puede mostrar tanto la aparición de potenciales anormalmente excesivos como en la epilepsia así como alteraciones bioeléctricas que ocasionan anormalidades en la distribución espacial de los ritmos eléctricos cerebrales, como en encefalopatías o disfunciones focales, ya sea por causas metabólicas o estructurales? 9

Los ritmos patológicos se pueden categorizar en: patrón rítmico periódico generalizado, que incluye descargas de ondas lentas, generalizadas, que se consideran de importancia clínica en pronóstico y evolución de pacientes con encefalopatía. Se refiere a descargas periódicas que son repetitivas, de < 0,5 segundos; con una morfología y duración consistentes de hasta 4 segundos ${ }^{10}$. El patrón rítmico no periódico generalizado incluye la actividad delta rítmica intermitente (IRDA) y actividad continua delta de alto voltaje ${ }^{11}$. La IRDA se presenta en pacientes con encefalopatía que se caracteriza por una serie de actividad rítmica de al to voltaje de 2 a $3 \mathrm{~Hz}$ que se repite a intervalos irregulares, sobre un fondo de disminución de theta generalizada de leve a moderada. Las ráfagas son sincrónicas y muy a menudo bilaterales, aunque pueden ser asimétricas o unilaterales ${ }^{7}$. Mientras que la actividad delta de alto voltaje asociada al coma es polimórfica, con una frecuencia de 1 a $2 \mathrm{~Hz}$, que refleja una etapa más profunda del coma que los patrones delta intermitentes u ondas trifásicas. Además, puede demostrar reactividad a la estimulación ${ }^{12}$. (Tabla 1 )

Se reviso los censos de la unidad de cuidados intensivos del hospital nacional Rosales, donde se encontró que en promedio hay 5-10 pacientes mensuales que se les diagnostica con encefalopatía, independiente del tipo. El hospital al momento no tiene protocolo de diagnóstico y atención inmediato para pacientes de esta índole, asociándose a altos índices de morbi-mortalidad, por lo que se pretende investigar la utilidad del EEG en el abordaje diagnóstico de la función cerebral, al registrar los distintos patrones electroencefalográficos en pacientes que son diagnosticados con encefalopatía, y correlacionarlos con su evolución clínica, para evidenciar si existe asociación con la evolución de los pacientes.

El objetivo principal del estudio fue presentar los patrones electroencefalográficos (EEG) y la evolución clínica de una serie de casos de pacientes con diagnóstico de encefalopatía, ingresados en la UCI (unidades médica y quirúrgica) del Hospital Nacional Rosales, de septiembre a diciembre de 2018 como una forma de contribuir al pronóstico.

\section{Metodología}

Se realizó estudio descriptivo de tipo serie de casos. Se incluyeron en forma consecutiva a todos los pacientes con diagnóstico de encefalopatía que ingresaron en las unidades de cuidados intensivos médicas y quirúrgicas del Hospital Nacional Rosales, durante los meses de septiembre a diciembre de 2018.

El estudio fue aprobado mediante acta de comité de ética de Hospital Nacional Rosales y la fuente de datos fueron los expedientes clínicos y reportes de electroencefalograma.

Se incluyeron 15 pacientes de ambos sexos, mayores de 12 años de edad, con diagnóstico de encefalopatía (hepática, metabólica, hipertensiva, isquémica, hipóxica, urémica, de Wernicke o cualquier otra) ingresados en las unidades de cuidados intensivos del Hospital Nacional Rosales, con estudio de EEG durante su estancia intrahospitalaria, para el análisis del patrón EEG y su relación con la evolución, que posea estudio de neuroimagen durante su estancia intrahospitalaria, para descartar que la alteración del estado de conciencia sea funcional y no estructural. Se aplicó proceso de consentimiento informado a los pacientes.

Se excluyeron pacientes que, previo al evento que causó la encefalopatía, presentaron enfermedad neurológica estructural como evento cerebrovascular, neoplasias, trauma craneoencefálico, hematoma subdural o lesiones estructurales de cráneo o cuero cabelludo que alteren el registro del ritmo electroencefalográficos, con anomalías estructurales encefálicas o defectos del cráneo o cuero cabelludo que no permitan la realización del electroencefalograma, como el trauma craneoencefálico abierto, 
craniectomizados, pacientes posteriores a drenaje de hematoma subdural, ventriculostomía, entre otros, y expedientes incompletos o sin registro EEG.

Se introdujeron todas las variables en una base de datos diseñada en el programa Microsoft Excel 2013. Se analizaron los datos en el programa estadístico Info v.7,2,1 y Software GraphPad Prism 6,0. Las variables de tipo cualitativo se analizaron según frecuencias y porcentajes. Para las variables de tipo cuantitativo, se determinó distribución; al no seguir una distribución normal se utilizaron medidas no paramétricas. Se calcularon medidas de tendencia central y medidas de dispersión, según tipo de variable.

\section{Resultados}

Se incluyeron 15 pacientes con diagnóstico de encefalopatía, a quienes se diferenció el tipo, comorbilidades y causa de ingreso a UCI (médica o quirúrgica). Según la distribución de pacientes, se observa que 14 fueron ingresados en la UCI médica y 1 en UCI quirúrgica. De ellos, 2 fallecieron en el periodo de tiempo que se encontraban en la UCI respectiva y 2 posterior a ser trasladados a otros servicios, para un total de mortalidad intrahospitalaria del 26,6 \%. La duración de estancia intrahospitalaria promedio fue de 29 días (rango de 3-77 días, con mediana de 21 días). La enfermedad de base identificada con más frecuencia en pacientes que desarrollaron como complicación encefalopatía fue la insuficiencia renal, siguiendo otras enfermedades crónicas (diabetes mellitus, hipertensión arterial, enfermedad pulmonar), enfermedades neuromusculares y epilepsia. La distribución por sexo fue $66,6 \%$ mujeres. La edad mínima fue de 15 años y el máximo de 84 años (mediana 41, rango intercuartílico de 26). El 93,3\% de los pacientes que ingresaron padecían de una enfermedad crónica; la más frecuente fue el diagnóstico de epilepsia (46,6\%), insuficiencia renal (33,3\%), hipertensión arterial (26,6 \%), diabetes mellitus y epilepsia (ambas con un $20 \%$ ) y alcoholismo crónico (13,3 $\%)$.

Un 71,4\% de pacientes con antecedentes de epilepsia presentaron encefalopatía epiléptica y el $100 \%$ de ellos presentó mejoría de la sintomatología (con tratamiento antiepiléptico), con un promedio de 21 días de estancia intrahospitalaria (rango entre 3 a 40 días).

Los pacientes que ingresaron con patologías neuromusculares (Guillain Barré y miastenia gravis) presentaron el mayor tiempo de estadía en la UCI, con un promedio de 60 días. En ambos se identificó hallazgos compatibles con encefalopatía de tipo hipóxico isquémica y complicaciones como neumonías nosocomiales.

En unidades de cuidados intensivos los pacientes ingresados tienden a presentar diversas complicaciones durante la estadía. En este estudio, el $80 \%$ de los pacientes presentó complicaciones durante el tiempo que estuvieron ingresados (Tabla 2). El $60 \%$ presentó neumonía, dividida entre nosocomial (40\%) y aspirativa (20\%).

La neumonía nosocomial fue la causa más frecuente de mortalidad, presentándose en el $75 \%$. Las úlceras por decúbito fueron la segunda más frecuente, presente en el $25 \%$, específicamente en pacientes cuya estadía fue mayor a 40 días ingresados.

El principal patrón electroencefalográfico que se encontró fue el patrón epileptiforme en el $60 \%$ de todos los pacientes ingresados (9 pacientes). De ellos, el $55 \%$ presentó encefalopatía epiléptica. Los patrones de ritmo periódico se presentaron en el $53 \%$ (8 pacientes), mientras que el no periódico en $46 \%$ (7 pacientes). El patrón brote supresión se identificó en una paciente con encefalopatía hipóxico isquémica.

El $53 \%$ de los pacientes presentaron más de un patrón electroencefalográfico, y fueron categorizados como patrones mixtos. El patrón epileptiforme, debido a su diversidad de presentaciones, se reflejó en el $50 \%$ de los patrones mixtos. Las encefalopatías metabólicas e hipóxico isquémicas estuvieron presentes en conjunto a otras ondas en el $20 \%$ de los pacientes cada una, encontrándose de forma predominante junto con la encefalopatía epileptiforme (Figura 1). 
De la muestra de 15 pacientes con diferentes tipos de encefalopatía, se identificaron dos pacientes (13\%) con encefalopatía metabólica de tipo osmótica. De ellos, uno presentó mejoría clínica y el otro permaneció en igual estado de conciencia durante los periodos de evaluación, que fueron entre 2 y 7 días de ingreso hospitalario. Dos pacientes $(13 \%)$ presentaron encefalopatía metabólica hiperglicémica, uno presentó mejoría clínica y uno falleció al quinto día de estancia intrahospitalaria. Cuatro pacientes (26\%) presentaron encefalopatía hipóxico isquémica, de los cuales dos permanecieron en igual estado de conciencia durante todo el estudio y dos pacientes fallecieron: uno por falla cardíaca y el otro por neumonía nosocomial. Solo un paciente $(6 \%)$ presentó encefalopatía hepática, en quién se observó mejoría del estado clínico.

Otro paciente $(6 \%)$ presentó encefalopatía urémica y falleció 38 días posterior a su ingreso. De los 15 pacientes del estudio, cinco (33\%) presentaron encefalopatía epiléptica; en cuatro se observó mejoría clínica y a un paciente se le dio alta hospitalaria.

Con respecto a la toma del EEG, al 53,3 \% de los pacientes se les tomó antes de las 12 horas desde su ingreso; al 13,3\% entre las $12-24$ horas y al $20 \%$ entre las $25-36$ horas posteriores al ingreso; al $13,3 \%$ restante durante los primeros 7 días.

Los patrones electroencefalográficos encontrados fueron principalmente patrones mixtos (variada actividad periódica, no periódica y/o epileptiforme), con $50 \%$. Posteriormente, patrón rítmico de ondas periódicas generalizadas con $25 \%$ y otro $25 \%$ con patrón rítmico de ondas no periódicas generalizadas.

Se identificó un patrón epileptiforme en el $66,6 \%$, con la peculiaridad de que este grupo presentó mejoría de estado de conciencia. Otros patrones fueron patrón ritmo periódico y patrón ritmo no periódico, que se comportaron de forma similar.

El $50 \%$ de los pacientes no presentó cambios en el estado de conciencia, el 33,3 \% presentó mejoría del estado de conciencia y el 13,6\% tuvieron empeoramiento del estado de conciencia. Solo un paciente presentó patrón brote supresión y uno con encefalopatía hipóxico isquémica, quien falleció en las primeras 48 horas de ingreso. De los pacientes que presentaron patrones mixtos, el $60 \%$ no tuvo cambios en el estado de conciencia en los seguimientos a las 48 horas y 7 días y el $40 \%$ restante sí presentó mejoría (Tabla 3).

Del total de pacientes el índice de mortalidad fue del $26 \%$; de ellos, el $25 \%$ falleció los primeros 5 días de estancia hospitalaria y el resto $(75 \%)$ posterior a 40 días de ingreso. Las causas principales de mortalidad fueron en $50 \%$ neumonía nosocomial como complicación durante su estancia hospitalaria. El tipo de encefalopatía que predominó en el $50 \%$ de pacientes que fallecieron fue la hipóxico isquémica grado 3 , seguida por un $25 \%$ con encefalopatía urémica y el $25 \%$ con metabólica osmótica; en ambos casos fue encefalopatía grado 2.

En cuanto a la reactividad del ritmo de fondo a maniobras de activación, durante la realización del EEG, se identificó que el $40 \%$ ( 6 pacientes) presentó reactividad en el patrón electroencefalográfico, mientras que el $60 \%$ (9) no. (Gráfica 1). Entre los pacientes con patrones electroencefalográficos con reactividad, el 83,3\% presentó mejoría durante las mediciones de tiempo 48 horas, 7 días y al alta, y el 26,6 \% restante no mostró cambios en el estado de consciencia. No se reportaron mortalidades de este grupo de pacientes. De los pacientes que no mostraron reactividad en los patrones electroencefalográficos, el 22,22 \% falleció previo al alta y el $22,22 \%$ posterior al alta, para un total de mortalidad de los pacientes que no presentaron reactividad del 44,44 .

\section{Discusión}

En las unidades de cuidados intensivos, el término encefalopatía implica un reto diagnóstico en los diferenciales de alteración del estado de conciencia, donde se debe indagar en el origen estructural o funcional del trastorno neurológico. Debido a que es una enfermedad multifactorial, requiere un abordaje integral basado en exploración clínica, pruebas neurofisiológicas (EEG, potenciales evocados) y neuroimagen. En ese contexto, el electroencefalograma es un método de bajo costo, accesible, que puede utilizarse para evaluar 
el estado neurológico de los pacientes ingresados en unidades de cuidados críticos, incluso a la cabecera del paciente 9 .

En pacientes críticamente enfermos, con cuadro clínico de encefalopatía o estados de reducción del estado de alerta hasta el coma, el análisis del registro electroencefalográfico provee información sobre disfunción cerebral, permite determinar la etiología de coma, diferenciando causas metabólicas entre causas tóxicas metabólicas de lesiones estructurales (por la lateralidad y focalidad de los hallazgos), determinar profundidad de estado de coma y para pronóstico clínico. Es útil, además, para seguimiento del curso de estado comatoso, ayuda a la diferencia del estado epiléptico no convulsivo y para monitoreo continuo de la función cerebral en unidad de cuidados críticos ${ }^{10}$. Este estudio identificó a pacientes con ausencia de alteración estructural, que tenían registro electroencefalográfico con disfunción córtico subcortical de diversa etiología y severidad.

En cuanto a edades, los rangos fueron amplios relacionados con el diagnóstico. En pacientes con diagnóstico de epilepsia, asociado a cambios encefalopáticos, el rango de edad fue menor, a diferencia de las encefalopatías de origen metabólico, que tuvieron un mayor rango de edad. Ramos J et al., de la Unidad de Neurología Pediátrica de España, encontró que el 80 \% de las encefalopatías epilépticas son dependientes de la edad ${ }^{13}$. Las encefalopatías hipóxico isquémicas son causadas por diferentes etiologías: en adultos mayores las causas principales son arrestos cardíacos y evento cerebro vascular y trauma craneoencefálico. Se describe que la media de los pacientes con estas patologías es arriba de los 60 años ${ }^{1}$. En esta serie de datos, que incluyó 15 pacientes, se identificó mayor frecuencia de mujeres, contrastando con datos obtenidos del Centro Nacional de Estadísticas en Salud de Estados Unidos, donde reportan con diagnóstico de encefalopatía 78 $\%$ masculino ${ }^{6}$.

El tiempo prolongado en las unidades de cuidados intensivos trae consigo una serie de posibles complicaciones. Merel $S$ describe en su artículo sobre las principales complicaciones en las unidades de cuidados intensivos, donde señala como una de las complicaciones más serias a la neumonía asociada a ventiladores y el deterioro cognitivo ${ }^{14}$. Esto coincide con el presente estudio, donde la principal patología que se encontró fue la neumonía nosocomial asociada a la ventilación mecánica, estando presente en el $60 \%$ de los pacientes ingresados. Como Kalanuria et al mencionan en su artículo sobre las neumonías asociadas a ventilador en la UCI y que se asocia con una elevada tasa de mortalidad. En ella describen que es totalmente prevenible y se propone el uso de un algoritmo preventivo, con factores como la elevación de la cabeza del paciente, cuidado bucal con lorhexidina, profilaxis trombótica, junto con sedación diaria y pruebas espontáneas de respiración ${ }^{15}$. La neumonía nosocomial asociada a ventilador presentó la mayor tasa de mortalidad de las complicaciones en UCI de esta serie, ya que el $50 \%$ de los pacientes que la presentaron fallecieron durante la estancia en UCI o al ser trasladados. En cuanto al tipo de patrón encefalográfico, el principal tipo de encefalopatía que estuvo presente en pacientes que fallecieron fue la metabólica. De 4 pacientes que fallecieron, 2 presentaron esta patología.

Berisavac I et al. describe en su estudio que las encefalopatías metabólicas varían en su severidad y estas dependen de su enfermedad de base. Las causas más comunes que ocasionan este tipo de encefalopatía son aquellas que provocan hipoxia, isquemia, enfermedades sistémicas y agentes tóxicos. Entre ellas se pueden mencionar: enfermedades cardiovasculares como insuficiencia cardíaca, hipertensión y arritmias, desbalances hidroelectrolíticos como la hiper e hipoglicemia, las sepsis y las enfermedades para neoplásicas ${ }^{16}$. Los pacientes del estudio que presentaron encefalopatías metabólicas y fallecieron presentaron diabetes mellitus descompensada, además de comorbilidades asociadas que también son vinculadas a las encefalopatías, como la hipertensión arterial y la sepsis.

Las descargas periódicas generalizadas fue el tipo más común de patrón epileptiforme que se encontró en los pacientes, reflejando la disfunción córtico subcortical global, asociado en este caso a causas funcionales, ya que a la mayoría de pacientes se le descartó causas estructurales que podrían haber generado descargas lateralizadas o focales. Este se describe como patrones en onda aguda, descargas polifásicas, onda lenta 
o agudizaciones, con duración y morfología constante. Estos pueden ser generalizados, lateralizados o focalizados en distribución, siendo las causas no estructurales más asociadas a descargas generalizadas, como en esta serie de datos. La información que se obtuvo de los pacientes de las UCI del HNR, la reactividad a maniobras de activación se asoció a pronóstico favorable y la no reactividad a mayor mortalidad, tal como se ha publicado en otras series.

Limitantes del estudio: es recomendable continuar la línea de investigación aplicando otro tipo de diseños con la finalidad de documentar un mayor número de casos que permitan establecer asociaciones entre los patrones electroencefalográficos y evolución clínica.

\section{CONCLUSIONES}

El diagnóstico basal más frecuente en pacientes que ingresaron con diagnóstico de encefalopatía fue epilepsia, asociado con cambios encefalopáticos y patrones con descargas epileptiformes generalizadas. Otras causas de encefalopatía fueron enfermedades metabólicas e hipoxia isquemia. El $80 \%$ de todos los pacientes ingresados en UCI presentaron complicaciones durante su estancia hospitalaria, siendo la más frecuente la neumonía nosocomial que se presentó en el $75 \%$ de los pacientes que fallecieron. Los patrones electroencefalográficos encontrados en los pacientes, clasificados con tipo y grado de encefalopatía, demuestran que la mortalidad está íntimamente relacionada con la severidad de la encefalopatía, así como con su progresión, duración, comorbilidades y complicaciones durante estancia hospitalaria. Los pacientes con reactividad no presentar fatalidades y sí mejoría en el estado de consciencia; mientras que los pacientes que no presentaron reactividad mostraron un alto porcentaje de mortalidad, pudiendo utilizar los patrones electroencefalográficos como una herramienta pronostica, que se podría demostrar en estudios de mayor profundidad.

\section{Conflicto DE INTERÉS}

Los autores no reportan ningún conflicto de interés.

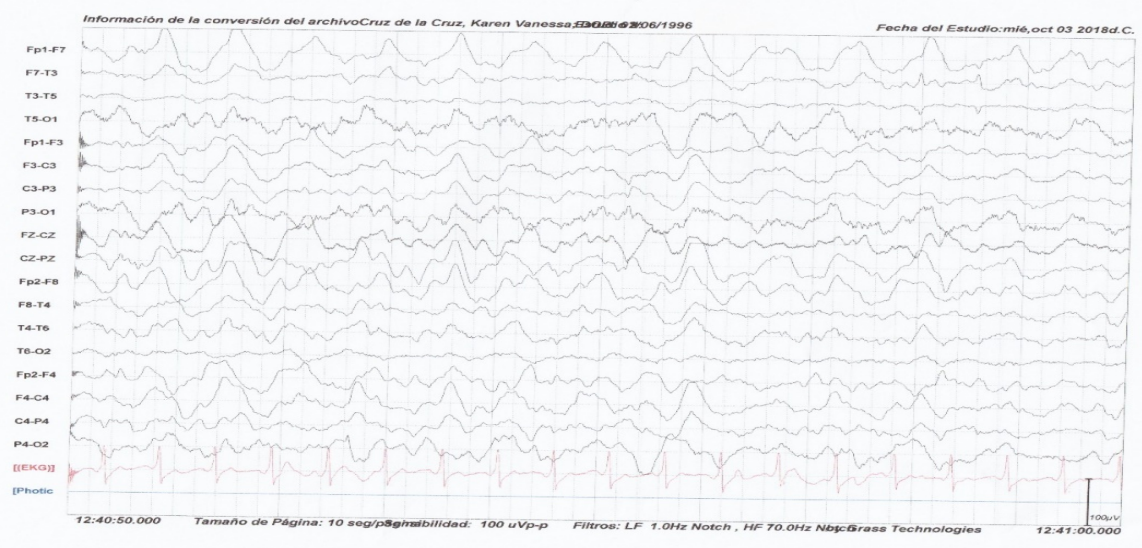

FIGURA 1

Paciente femenina de 15 años que ingresó a UCI médica, con diagnóstico de estatus epiléptico y con sospecha de Neuro lupus. En EEG se identifica actividad epiléptica generalizada y patrón rítmico periódico bilateral independiente y onda aguda y punta lenta de predominio frontal Elaboración propia a partir de los datos recopilados 
TABLA 1

Categorización de patrones electroencefalográficos

\begin{tabular}{|c|c|c|}
\hline Terminología 1 & Terminología 2 & Modificadores \\
\hline G & PD & Prevalencia \\
\hline \multirow{9}{*}{$\begin{array}{l}\text { Generalizado: específico, frontal, línea } \\
\text { media u occipitalmente predominante }\end{array}$} & Descargas periódicas & Duración \\
\hline & $\begin{array}{l}\text { Un tipo de descargas repetitivas con } \\
\text { un intervalo de descarga cuantitativa } \\
\text { cuantificable y recurrencia a intervalos casi } \\
\text { regulares. Es un tipo de onda que dura }<4 \\
\text { segundos independientemente del número } \\
\text { de fases }\end{array}$ & Frecuencia \\
\hline & & Número de fases \\
\hline & & Ondas \\
\hline & & Amplitud \\
\hline & & Polaridad \\
\hline & & Reactividad \\
\hline & & Evolucionando o \\
\hline & & Fluctuando \\
\hline $\mathrm{L}$ & RDA & $\begin{array}{l}\text { + Se refiere a características que sugieren } \\
\text { que un patrón periódico puede ser más } \\
\text { ictal }\end{array}$ \\
\hline $\begin{array}{l}\text { Lateralizado: específicamente unilateral o } \\
\text { bilateral asimétrico }\end{array}$ & Actividad Delta rítmica & \\
\hline $\begin{array}{l}\text { Opcional: lóbulo específico involucrado o } \\
\text { hemisferio }\end{array}$ & $\begin{array}{l}\text { Repetición de una forma de onda con una } \\
\text { morfología y duración uniformes, cuya } \\
\text { frecuencia es de } 4 \mathrm{~Hz}\end{array}$ & \\
\hline $\mathrm{BI}$ & SW & $\mathrm{F}$ \\
\hline Bilateral independiente & $\begin{array}{l}\text { Onda y punta rítmica o polipunta y onda } \\
\text { rítmica }\end{array}$ & $\begin{array}{l}\text { Aplica tanto para PD como para RDA, } \\
\text { indica una actividad rápida superpuesta. }\end{array}$ \\
\hline Especificamente simétrico o asimétrico & $\begin{array}{l}\text { Se refiere a una onda aguda con una onda } \\
\text { lenta que se produce en un patrón que se } \\
\text { repite regularmente sin ningún intervalo } \\
\text { entre si. (Si hay un intervalo sería PD) }\end{array}$ & $\mathrm{R}$ \\
\hline \multirow[t]{3}{*}{$\begin{array}{l}\text { Opcional: lóbulo específico involucrado o } \\
\text { hemisferio }\end{array}$} & & $\begin{array}{l}\text { Aplica solo a las PD e indica actividad } \\
\text { Delta rítmica superpuesta }\end{array}$ \\
\hline & & $\mathrm{S}$ \\
\hline & & $\begin{array}{l}\text { Aplica solo a RDA e indica ondas o } \\
\text { puntas afiladas superpuestas o actividad } \\
\text { contorneada aguda }\end{array}$ \\
\hline Mf & & $\mathrm{FR}$ \\
\hline Multifocal & & Para descargas periódicas \\
\hline $\mathrm{BI}$ & & FS \\
\hline Bilateral independiente. & & Para RDA \\
\hline \multicolumn{3}{|l|}{ Específicamente simétrico o asimétrico } \\
\hline $\begin{array}{l}\text { Opcional: lóbulo específico involucrado o } \\
\text { hemisferio }\end{array}$ & & \\
\hline
\end{tabular}

Hirsch LJ, et al. American Clinical Neurophysiology Society's standardized critical care EEG terminology: 2012 version. J Clin Neurophysiol 2013; 30(1):1-27 
TABLA 2

Complicaciones en pacientes ingresados en UCI, según mortalidad o supervivencia

\begin{tabular}{lcr}
\hline \multicolumn{1}{c}{ Complicaciones } & Defunción & Supervivencia \\
\hline Paro cardiorespiratorio & 1 & 0 \\
Shock Séptico & 1 & 2 \\
Neumonía nosocomial & 3 & 3 \\
Neumonía aspirativa & 0 & 3 \\
Úlceras por decúbito & 1 & 2 \\
Desequilibrio hidroelectrolítico & 0 & 3 \\
\hline
\end{tabular}

Elaboración propia a partir de los datos recopilados 
TABLA 3

Complicaciones en pacientes ingresados en UCI, según mortalidad o supervivencia

\begin{tabular}{|c|c|c|c|c|c|c|c|c|c|c|c|}
\hline Código & Edad & Sexo & $\begin{array}{l}\text { Día en } \\
\text { UCI }\end{array}$ & $\begin{array}{l}\text { Diagnóstico de } \\
\text { ingreso a UCl }\end{array}$ & $\begin{array}{c}\text { Tipo de } \\
\text { Encefalopatía }\end{array}$ & $\begin{array}{l}\text { Tipo de Patrón } \\
\text { electroencefalográfico }\end{array}$ & Reactividad & Simetria & Defunción & $\begin{array}{c}\text { Grado de } \\
\text { severidad de } \\
\text { Encefalopatía }\end{array}$ & Uso de sedantes \\
\hline 1 & 55 & $\mathrm{~F}$ & 43 días & $\begin{array}{l}\text { Insuficiencia } \\
\text { Respiratoria }\end{array}$ & $\begin{array}{l}\text { Hipóxico } \\
\text { isquémica }\end{array}$ & $\begin{array}{c}\text { Patrón epileptiforme/ } \\
\text { Patrón Brote } \\
\text { Supresión/ Patrón } \\
\text { mixto }\end{array}$ & NO & Sí & Sí & Grado 3 & Benzodiacepinas \\
\hline 2 & 30 & M & 40 dias & $\begin{array}{l}\text { Estado } \\
\text { Epiléptico }\end{array}$ & Metabólica & $\begin{array}{l}\text { Patrón epileptiforme/ } \\
\text { Patrón de ondas } \\
\text { agudas/ Patrón mixto }\end{array}$ & NO & NO & & Grado 2 & Otros \\
\hline 3 & 27 & $\mathrm{~F}$ & 69 días & $\begin{array}{l}\text { Insuficiencia } \\
\text { Renal }\end{array}$ & Urémica & Patrón No Periódico & NO & NO & SÍ (UCI) & Grado 2 & Otros \\
\hline 4 & 70 & $\mathrm{~F}$ & 21 días & $\begin{array}{l}\text { Insuficiencia } \\
\text { Respiratoria }\end{array}$ & Metabólica & Patrón Periódico & NO & NO & Sí & Grado 2 & Benzodiacepinas \\
\hline 5 & 15 & $\mathrm{~F}$ & 3 días & $\begin{array}{l}\text { Estado } \\
\text { Epiléptico }\end{array}$ & Epiléptica & $\begin{array}{l}\text { Patrón Periódico/ } \\
\text { Patrón epileptiforme/ } \\
\text { Patrón mixto }\end{array}$ & NO & NO & & Grado 2 & \\
\hline 6 & 40 & $\mathrm{~F}$ & 63 días & $\begin{array}{l}\text { Insuficiencia } \\
\text { Renal }\end{array}$ & Metabólica & $\begin{array}{l}\text { Patrón No Periódico/ } \\
\text { Patrón Epileptiforme/ } \\
\text { Patron Mixto }\end{array}$ & NO & Sí & & Grado 3 & \\
\hline 7 & 84 & M & 6 días & $\begin{array}{l}\text { Diabetes } \\
\text { Mellitus } \\
\text { Descompensada }\end{array}$ & Metabólica & Patrón Periódico & Sí & Sí & & Grado 3 & \\
\hline 8 & 61 & $\mathrm{~F}$ & 4 días & $\begin{array}{l}\text { Diabetes } \\
\text { Mellitus } \\
\text { Descompensada }\end{array}$ & $\begin{array}{l}\text { Hipóxico } \\
\text { isquémica }\end{array}$ & Patrón Periodico & NO & NO & SÍ (UCI) & Grado 3 & Benzodiacepinas \\
\hline 9 & 50 & M & 38 días & $\begin{array}{l}\text { Estado } \\
\text { Epiléptico }\end{array}$ & Epiléptica & $\begin{array}{l}\text { Patrón Periódico/ } \\
\text { Patrón Epileptiforme/ } \\
\text { Patrón mixto }\end{array}$ & Sí & NO & & Grado 2 & Benzodiacepinas \\
\hline 10 & 19 & M & 6 días & $\begin{array}{l}\text { Estado } \\
\text { Epiléptico }\end{array}$ & Hepática & Patrón Epileptiforme & Sí & Sí & & Grado 3 & Benzodiacepinas \\
\hline 11 & 61 & $\mathrm{~F}$ & 77 días & Neumonía & $\begin{array}{l}\text { Hipóxico } \\
\text { isquémica }\end{array}$ & Patron no periodico & Sí & NO & & Grado 3 & \\
\hline 12 & 22 & $\mathrm{~F}$ & 15 dias & $\begin{array}{l}\text { Estado } \\
\text { Epiléptico }\end{array}$ & Epiléptica & Patron No Periodico & NO & Sí & & Grado 1 & Otros \\
\hline 13 & 26 & $\mathrm{~F}$ & 19 días & $\begin{array}{l}\text { Estado } \\
\text { Epiléptico }\end{array}$ & Epiléptica & $\begin{array}{l}\text { Patrón Periódico/ } \\
\text { Patrón Epileptiforme } \\
\text { / Patrón Mixto }\end{array}$ & NO & Sí & & Grado 3 & Benzodiacepinas \\
\hline 14 & 41 & M & 13 días & $\begin{array}{l}\text { Insuficiencia } \\
\text { Respiratoria }\end{array}$ & $\begin{array}{l}\text { Hipóxico } \\
\text { isquémica }\end{array}$ & $\begin{array}{l}\text { Patron No Periódico/ } \\
\text { Patron epileptiforme/ } \\
\text { Patron Mixto }\end{array}$ & Sí & Sí & & Grado 2 & \\
\hline 15 & 51 & $\mathrm{~F}$ & 23 días & $\begin{array}{l}\text { Estado } \\
\text { Epiléptico }\end{array}$ & Epiléptica & $\begin{array}{l}\text { Patrón no periódico/ } \\
\text { Patrón epileptiforme/ } \\
\text { Patrón mixto }\end{array}$ & SÍ & SÍ & & Grado 1 & Benzodiacepinas \\
\hline
\end{tabular}

Patrones electroencefalográficos y evolución clínica en pacientes con diagnóstico de encefalopatía, ingresados en unidades de cuidados intensivos (Médica y Quirúrgica) del Hospital Nacional Rosales, de septiembre a diciembre de 2018

\section{REFERENCIAS BIBLIOGRÁFICAS}

1. Encephalopathy| National Institute of Neurological Disorders and Stroke [Internet]. Ninds.nih.gov. 2018 [cited 16 August 2018]. Available from: https://www.ninds.nih.gov/Disorders/All-Disorders/Encephalopathy-Infor mation-Page

2. Elwir S, Rahimi RS. Hepatic Encephalopathy: An Update on the Pathophysiology and Therapeutic Options. Journal of Clinical and Translational Hepatology. 2017; 5(2):142-151. DOI: 10.14218/JCTH.2016.00069. Available from: https://www.ncbi.nlm.nih.gov/pmc/articles/PMC5472935/

3. Castellanos Pinedo F, Cid-Gala M, Duque P., et al. Daño cerebral sobrevenido: propuesta de definición, criterios diagnósticos y clasificación. Rev Neurol 2012; 54 (06):357-366

4. NAMCS/NHAMCS - Ambulatory Health Care Data Homepage [Internet]. Cdc.gov. 2019 [cited 23 February 2019]. Available from: https://www.cdc.gov/nchs/ahcd/index.htm 
5. Romero M, et al. Encefalopatía hepática, clasificación y manifestaciones clínicas. Unidad de Hepatología. Sevilla España GH continuada May/jun 2004. Vol. 3.

6. Bauer G. EEG patterns in hypoxic encephalopathies (post cardiac arrest syndrome): fluctuations, transitions and reactions. Journal of Clinical Neurophysiology. 2013; 30(5): 477-89.

7. Martinez B, et al. Encefalopatías metabólicas. Servicio de neurología pediátrica. Asociación española de pediatría. 2008.

8. Nicholas D, et al. Multifocal periodic lateralized epileptiform discharges (PLEDs): EEG features and clinical correlations. Clinical Neurophysiology 111 (2000) 2125-2129.

9. Freund B. and Kaplan P. W. Systematic review of bilateral independent periodic discharges written for tropical journal subject on periodic discharges. Journal of clinical Neurophysiology. 2018; 35(3):208-217.

10. Cosenza A. M., Andraus S. F. Alves-León S., Periodic EEG patterns: Importance of their recognition and clinical significance. Arq. Neuropsiquiatr. 2012; 70(2): 145-151.

11. Sarkis RA, Lee JW. Quantitative EEG in hospital encephalopathy: review and microstate analysis. [Internet]. Advances in paediatrics. U.S. National Library of Medicine; 2013. Disponible en: https://www.ncbi.nlm.nih. gov/pubmed/24084185

12. Ramos R, Rodríguez L. Review of the final report of the 1998 working party on definition, nomenclature and diagnosis of hepatic encephalopathy. Ann Hepatol. 2011; 10(2):s36-s39.

13. Asabou E, et al., Value and mechanism of EEG reactivity in the prognosis of Patients with impaired consciousness: A systematic review. Crit Care. Aug. 2018. 2; 22 (1):184.

14. Merel S. Complications of intensive care unit care [Internet]. Cancer Therapy Advisor. 2017 [cited 23 February 2019]. Available from: https://www.cancertherapyadvisor.com/hospital-medicine/complications-of-intensive -care-unit-care/article/602609/

15. Kalanuria A, Zai W, Mirski M. Ventilator-associated pneumonia in the ICU. Critical Care. 2014; 18(2):208.

16. Berisavac I, Jovanović D, Padjen V, Ercegovac M, Stanarčević P, Budimkić-Stefanović M, et al. How to recognize and treat metabolic encephalopathy in Neurology intensive care unit. Neurology India. 2017; 65(1):123. 